\title{
12. Generationsübergreifende Auswirkungen von Traumata: Implikationen für Individuen und Gesellschaft
}

Aus dem Englischen übersetzt von Anja Pichl und Lilian Marx-Stölting

Lebenserfahrungen sind wichtige Einflussfaktoren auf unser Verhalten. Während positive Erfahrungen, wie z. B. eine liebevolle Familie, ein stressfreies Arbeitsumfeld und harmonische soziale Interaktionen, förderlich sind, können negative Erfahrungen, wie z. B. schlechte Lebensbedingungen und traumatische Ereignisse, die Lebensqualität beeinträchtigen. Insbesondere ein psychologisches Trauma, definiert als überwältigender Stress, der also über die Fähigkeit eines Individuums, ihn zu bewältigen, hinausgeht, kann lang anhaltende Auswirkungen auf die psychische und physische Gesundheit haben. Solche Erfahrungen wirken sich auf die exponierten (ihm ausgesetzten) Individuen aus und erhöhen ihre Anfälligkeit für Krankheiten. Sie können sich auch auf ihre Nachkommen auswirken, in manchen Fällen über mehrere Generationen hinweg, auch wenn diese Nachkommen selbst keinem Trauma ausgesetzt waren (Jawaid/Mansuy, 2021; Jawaid et al., 2018). Diese Art der Vererbung stellt das herkömmliche Konzept der Vererbung in Frage, bei dem davon ausgegangen wird, dass nur genetische Faktoren einzubeziehen sind. Stattdessen hängt die Übertragung nicht von der DNA-Sequenz ab, die als genetischer Code dient, sondern impliziert Mechanismen wie bestimmte Signalwege, die die Aktivität des genetischen Codes verändern, ohne die Sequenz zu verändern; dies ist als Epigenetik bekannt (siehe Walter/Gasparoni, Kap. 3). Diese Mechanismen werden in Reaktion auf verschiedene Umwelteinflüsse aktiviert. Ihre Veränderung kann stabil an die Nachkommen weitergegeben werden und zu bestimmten Merkmalen bei diesen Nachkommen führen, mitunter über Generationen hinweg (Bohacek/Mansuy, 2015). Diese Form der Übertragung wird als transgenerationale epigenetische Vererbung bezeichnet, wenn sie Individuen über mehrere Generationen hinweg betrifft, und als intergenerationale epigenetische Vererbung, wenn sie nur die direkten Nachkommen betrifft. 
Während die Idee, dass durch psychische Traumata hervorgerufene Symptome vererbbar sind, schon lange als Hypothese im Raum stand, ist die biologische Basis ihrer langfristigen und potenziell vererbbaren Auswirkungen erst seit Kurzem bekannt. Traumatische Erfahrungen (Traumaexpositionen) können in der Kindheit oder im späteren Leben auftreten, z. B. in Form von körperlichem oder sexuellem Missbrauch, entzogener elterlicher Fürsorge, Naturkatastrophen oder erzwungener Vertreibung, und können zusätzliche Faktoren wie das Erleben militärischer Kämpfe im Erwachsenenalter umfassen (Lupien et al., 2009). Diese Erfahrungen, sowohl während der Kindheit als auch im Erwachsenenalter, sind wichtige Risikofaktoren für psychische Störungen, können aber auch die körperliche Gesundheit beeinflussen. Eine schwerwiegende Folge psychischer Traumata beim Menschen ist die Entwicklung einer posttraumatischen Belastungsstörung (PTBS), die häufig mit Depressionen und Angstzuständen einhergeht (Ginzburg et al., 2010) und mit einer größeren Häufigkeit (Prävalenz und Inzidenz) ${ }^{1}$ von Demenz assoziiert wird (Qureshi et al., 2010). Abgesehen vom Gehirn hat chronisch traumatischer Stress auch schädliche Auswirkungen auf andere Körperfunktionen. PTBS ist ein Risikofaktor für kardiovaskuläre (den Kreislauf und die Blutgefäße betreffende) und zerebrovaskuläre (die Blutgefäße des Gehrins betreffende) Erkrankungen, Störungen des Verdauungssystems (gastrointestinale Dysfunktionen), rheumatische Arthritis und Krebs (Qureshi et al., 2010). Wichtig ist, dass ein Trauma eine Reihe von Signalwegen aktivieren kann, die die Auswirkungen des Traumas auf die Fortpflanzungsorgane und die Keimbahn übertragen können. Das bedeutet, dass sie vererbbar sein können. Folglich könnten Veränderungen in verschiedenen Geweben, Organen, Körperflüssigkeiten und pathophysiologischen Signalwegen nach einem Trauma solch eine Übertragung anstoßen (Jawaid et al., 2021).

Die Vorstellung, dass psychologische Traumata zu Effekten führen, die potenziell vererbbar sind, ist von großer Bedeutung für die Gesellschaft, wenn man bedenkt, wie viele Individuen durch gegenwärtige und jüngste menschliche Konflikte traumatisiert wurden. Dieses Kapitel diskutiert das Konzept der epigenetischen Vererbung im Zusammenhang mit Merkmalen, die aus einer solchen Traumaexposition resultieren, sowie deren Auswirkungen auf das Leben eines Individuums und auf die Gesellschaft. Es gibt einen Überblick über Studien an Tieren und Menschen zur inter- und transgenerationalen Weitergabe der Auswirkungen psychologischer Traumaexpositionen, indem es die Frage behandelt, wie Traumaexposition und damit verbundene emotionale und kognitive Störungen Spuren in der Keimbahn hinterlassen können. Auch die Frage

1 Die Inzidenz bezieht sich auf die Anzahl von Neuerkrankungen, die Prävalenz gibt hingegen die Anzahl der Neuerkrankungen geteilt durch die Anzahl der Individuen einer Population an. 
nach "Gelegenheitsfenstern“ in verschiedenen Lebensabschnitten, von der Kindheit bis zum Erwachsenenalter, in denen die Vererbung von Traumafolgen verhindert werden kann, wird diskutiert. Insbesondere arbeitet das Kapitel die Implikationen der Forschung über die Vererbung von Traumafolgen im Zusammenhang mit politischen und ethnischen Konflikten heraus. Dabei geht es auch darum, was Menschen, die langfristig Frieden sichern wollen (,peace builder"), aus dem Gebiet der epigenetischen Vererbung lernen können und wie sie mit Wissenschaftler*innen zusammenarbeiten können, um Strategien und politische Entscheidungen mit biologischen Erkenntnissen zu bereichern und den Frieden zu fördern (Jawaid/Mansuy, 2021).

\subsection{Einführung in die Epigenetik}

Die Epigenetik ist ein Zweig der Biologie, der sich mit vererbbaren Veränderungen beschäftigt, die keine Veränderung der DNA-Sequenz beinhalten (siehe Walter/Gasparoni, Kap. 3). Man geht davon aus, dass diese Veränderungen durch eine Kombination aus chemischen Modifikationen der DNA (z. B. Hinzufügen von Methylmolekülen; DNA-Methylierung), ähnlichen Modifikationen von Histonen und Proteinen, die DNA-Moleküle umgeben, sowie RNA-Molekülen, ${ }^{2}$ die den in DNA-Sequenzen vorhandenen genetischen Code weitergeben, übertragen werden (Bohacek/Mansuy, 2015).

\subsubsection{DNA-Methylierung}

DNA-Methylierung ist ein Prozess, bei dem Methylgruppen an DNA-Moleküle angehängt werden, die verhindern, dass die DNA-Expressionsmaschinerie ${ }^{3}$ an die DNA binden kann. Sie wird typischerweise mit Gene-Silencing in Verbindung gebracht, also mit der Stilllegung von Genen, die folglich nicht mehr abgelesen werden (Bird, 2002; Klose/ Bird, 2006). Gelegentlich und unter bestimmten Bedingungen kann die Methylierung jedoch auch die Genexpression fördern (Bahar Halpern et al., 2014). Die DNA wird durch komplexe Mechanismen in Reaktion auf verschiedene Expositionen dynamisch methyliert und demethyliert, was manchmal zu vorübergehend auftretenden Zwischen-

2 Histone sind die Proteine, um die die DNA im Chromosom aufgewickelt ist; Proteine, die DNAMoleküle umgeben sind beispielsweise Transkriptionsfaktoren, die das Ablesen der DNA beeinflussen. RNA-Moleküle sind Nukleinsäuren, die im Prozess der Transkription entstehen, wenn die DNA abgelesen wird. Sie können selbst Aufgaben in Zellen übernehmen oder als Vorlage für die Proteinbiosynthese im Prozess der Translation dienen. Genauere Erläuterungen siehe im Text weiter unten.

3 Zur Expressionsmaschinerie gehören verschiedene Proteine, die an die DNA andocken müssen, um diese in RNA umschreiben, also transkribieren zu können. Die Methylierung der DNA verhindert, dass sich der Proteinkomplex bilden kann. 
formen führt, die ebenfalls unterschiedliche Auswirkungen haben können. Neuere Forschungen haben gezeigt, dass die Traumaexposition die DNA-Methylierung beeinflussen kann, sowohl generell für alle Gene als auch manchmal für einige spezifische Gene (Jawahar et al., 2015), was sie zu einem möglichen Mechanismus für langfristige und möglicherweise vererbte Auswirkungen von Traumata macht (Jawaid et al., 2021).

\subsubsection{Histon-Modifikationen}

Histone sind DNA-assoziierte Proteine, die die DNA umgeben und sie im Chromosom in einer fest verpackten, stillen (also nicht ablesbaren) Form halten können. Verschiedene Moleküle können jedoch die Affinität der Histonmoleküle zur DNA modifizieren und damit die Zugänglichkeit der DNA-Expressionsmaschinerie zur DNA verändern (Bannister/Kouzarides, 2011). Zu diesen Modifikationen gehört das Anhängen bestimmter Gruppen an die Histone: die Acetylierung (Anfügung einer Acetylgruppe), die Methylierung (Anfügung einer Methylgruppe), die Phosphorylierung (Anfügung einer Phosphorylgruppe), die Ubiquitinierung (Anfügung eines universellen Ubiquitin-Proteins) und die SUMOylierung (Anfügung von kleinen Ubiquitin-ähnlichen Modifikatoren) (Li et al., 2007). Einige dieser Modifikationen öffnen die Histone, was die Genexpression begünstigt, während andere das Histongeflecht um die DNA noch enger machen und dadurch die Genexpression verhindern (Peters/Schübeler, 2005; Cao/Yan, 2012; Cheng et al., 2014). Auch hier hat sich in Tiermodellen gezeigt, dass traumatischer Stress die Histone modifiziert, die die für Verhaltensreaktionen wichtigen Gene umgeben (Hunter et al., 2009; Reed et al., 2012). Präklinische Studien, die den Einsatz von HistonDeacetylase(HDAC)-Inhibitoren, die die Histon-Acetylierung verstärken, in Traumaund Angsttherapien nahelegen, liefern weitere Belege für die Annahme einer Rolle der Histonregulation während und nach traumatischem Stress (Whittle/Singewald, 2014).

\subsubsection{RNA}

RNA-Moleküle übertragen den genetischen Code, der in der Basensequenz der DNAMoleküle vorhanden ist, auf Proteine. Jede Störung in diesem Prozess bietet eine weitere Möglichkeit, die Genexpression verändern, ohne den genetischen Code zu verändern. Die RNA-Moleküle, die die von der DNA codierte Information übertragen, werden als codierende RNAs bezeichnet, die durch einige andere RNAs, die keine genetische Information übertragen, reguliert werden können. Zwei Klassen von nicht-kodierenden RNAs (ncRNAs), kleine und lange ncRNAs, sind hier besonders relevant. Unter den kleinen RNAs wirken microRNAs (miRNAs) durch Abbau oder Unterdrückung von Boten- 
RNAs („messenger RNAs“, mRNAs), die direkt von der DNA auf Basis des genetischen Codes produziert werden (Ha/Kim, 2014). Dagegen können lange ncRNAs (lncRNAs) die Funktionen von miRNAs stören und mit ihnen konkurrieren. Sie können auch die mRNA-Stabilität und -Prozessierung beeinflussen (Quinn/Chang, 2016). Sowohl miRNAs als auch lncRNAs wurden bei Versuchsmäusen mit der Übertragung der Langzeiteffekte einer Traumaexposition in Verbindung gebracht (Jawaid et al., 2021). Darüber hinaus haben Studien an PTBS-Patient*innen eine mögliche Beteiligung von miRNAs als Mediatoren und/oder Moderatoren der Auswirkungen von traumatischem Stress aufgezeigt (Snijders et al., 2017).

Alle diese epigenetischen Mechanismen wirken in verschiedenen Zellen des Körpers, einschließlich der Keimzellen, die auf die Fortpflanzung spezialisiert sind, wie Spermien und Eizellen. Die Aktivierung epigenetischer Signalwege in solchen Zellen könnte daher an der Übertragung der Auswirkungen eines Traumas auf die Nachkommenschaft beteiligt sein. Im folgenden Abschnitt erörtern wir die epigenetischen Faktoren, von denen bekannt ist, dass sie durch traumatische oder stressige Expositionen in der Keimbahn modifiziert werden, und diskutieren die Bedeutung dieser Erkenntnisse.

\subsection{Epigenetische Mechanismen in der Keimbahn}

Ein frühes Lebenstrauma verändert bei Mäusen das Niveau der DNA-Methylierung von Genen, die an für die Stressregulation und kognitive Verarbeitung wichtigen Signalkaskaden beteiligt sind. Ähnliche Veränderungen in der DNA-Methylierung sind auch im Gehirn und im Sperma der Nachkommen vorhanden (Franklin et al., 2010), was darauf hindeutet, dass sie vom Vater auf die Nachkommen übertragen wurden.

Ebenso ist aus der Forschung an D. melanogaster (Fruchtfliege) und C. elegans (Regenwurm) bekannt, dass Umweltstressfaktoren chemische Modifikationen von Histonen und verwandten Proteinen verändern (Gaydos et al., 2014; Ciabrelli et al., 2017). Bei Nagetieren und Menschen werden jedoch die meisten Histone im Sperma durch eine andere Klasse von Proteinen ersetzt (Protamine genannt), weshalb der Beitrag der Histone zur epigenetischen Vererbung begrenzt sein könnte (Bohacek/Mansuy, 2015).

Heute ist RNA der wichtigste Faktor, dem eine kausale Rolle bei der Übertragung von erworbenen Merkmalen zugeschrieben wird. Spermien-RNA ist an der Übertragung von traumatischem Stress vom Vater auf die Nachkommenschaft beteiligt (Gapp et al., 2014a und 2020). So kann die Injektion von Spermien-RNA männlicher Mäuse, die im postnatalen Leben einem Trauma ausgesetzt waren, in befruchtete Kontrolleizellen die Verhaltens- und Stoffwechselsymptome bei den daraus entstehenden Mäusen und 
ihren Nachkommen nachbilden. In einigen Fällen kann die Injektion von 9 miRNAs, von denen bekannt ist, dass sie in Spermien durch adulten väterlichen Stress hochreguliert werden, den Spiegel des Stresshormons Corticosteron in den resultierenden Tieren verändern (Rodgers et al., 2015). Des Weiteren wurde gezeigt, dass Spermien-miRNAs für die Auswirkung von Environmental Enrichment (die Stimulierung des Gehirns durch die physische und soziale Umgebung) auf die Verbesserung der Kognition verantwortlich sind (Benito et al., 2018). Von diesem Beispiel ist auch bekannt, dass es die Übertragung einiger Auswirkungen der Traumata bei Mäusen verhindert (Gapp et al., 2016). Somit könnte die Spermien-RNA ein gemeinsamer Faktor für die Übertragung positiver und negativer Effekte von Lebenserfahrungen sein, ein Mechanismus, der neue Perspektiven für die Vererbung bietet.

\subsection{Vererbung von Traumafolgen in Tiermodellen und beim Menschen}

Es gibt immer mehr Belege dafür, dass die Auswirkungen von Trauma und Widrigkeiten in Tiermodellen auf nachfolgende Generationen übertragen werden können und die Keimbahn betreffen. Es ist wichtig zu berücksichtigen, dass die intergenerationale Übertragung der Auswirkungen von Trauma unabhängig von der Keimbahn sein kann und eine soziale Übertragung und der Erwerb von Verhaltens- oder physiologischen Mustern von den Eltern auf die Nachkommen möglich ist. Diese können jedoch epigenetische Faktoren z. B. im Gehirn einbeziehen. Aber die echte Vererbung der Auswirkungen von traumatischem Stress beinhaltet die Übertragung durch Keimzellen und impliziert epigenetische Mechanismen (Bohacek/Mansuy, 2015).

Bei Nagetieren beeinflusst ein Trauma das Verhalten und den Stoffwechsel nachfolgender Generationen durch komplexe epigenetische Mechanismen, die nur teilweise verstanden sind. Ähnliche Effekte können auch beim Menschen auftreten, aber dafür gibt es weniger Belege und das Wissen über involvierte Mechanismen ist sehr begrenzt. Der folgende Abschnitt fasst einschlägige Beweise (Evidenz) für die inter- und transgenerationale Vererbung der Auswirkungen von Traumata bei Tieren und Menschen zusammen.

\subsubsection{Nagetiermodelle}

In Studien zur Vererbung, z. B. zu Traumaeffekten, wird typischerweise eine Nomenklatur verwendet, bei der die Nachkommen, die aus der ersten genetischen Kreuzung hervorgehen, als F1 (filial/Nachkommen 1) bezeichnet werden und nachfolgende Ge- 
nerationen, die die Paarung von betroffenen (Trauma) F1 mit naiven Züchtungen oder betroffenen F1 beinhalten, als F2, F3 usw. In Tierversuchen wurde Trauma typischerweise durch die Störung oder Unterbrechung der mütterlichen Fürsorge oder durch die Konditionierung der Tiere auf unangenehme oder furchterregende Reize ausgelöst.

Bei Mäusen wurde eine Veränderung der mütterlichen Fürsorge durch Stress für das Muttertier während der Laktation mit einer verminderten sozialen Interaktion bei den Nachkommen in Verbindung gebracht, die auf eine suboptimale mütterliche Fürsorge zurückgeführt wird. Zusätzlich zu den Verhaltensanomalien wiesen die männlichen Nachkommen auch Veränderungen von Oxytocin und Corticosteron auf, zwei Hormonen, die in die Regulierung von Stress und sozialer Bindung bei Säugetieren involviert sind, während die weiblichen Nachkommen verändertes Prolaktin aufwiesen, ein Hormon, das für die Laktation wichtig ist (Babb et al., 2014). In ähnlicher Weise war die Auslösung einer postpartalen Depression (Wochenbettdepression) bei stillenden Müttern durch tagsüber gegenwärtige Stressfaktoren (,daily restraint“) und nächtliche Beleuchtung mit der Vererbung von depressiven Verhaltensweisen und veränderten Stoffwechselwegen bei den F2-Nachkommen verbunden (Wu et al., 2016).

Unsere Versuchsanordnung der unvorhersehbaren Trennung von der Mutter (,unpredictable maternal separation") in Kombination mit unvorhersehbarem mütterlichen Stress (,unpredictable maternal stress“ [MSUS]) ist eines der besten Modelle für transgenerationale Vererbung bei Mäusen (Franklin et al., 2010). Dieses Modell zeigt eine klare Keimbahnabhängigkeit, eine Ursache-Wirkungs-Beziehung und betrifft bis zu vier aufeinanderfolgende Nachkommengenerationen (van Steenwyk et al., 2018). Bei diesem Paradigma werden neugeborene Mäusejunge (F1) traumatischem Stress ausgesetzt, indem sie unvorhersehbar von ihrer Mutter (Fo) getrennt werden. Während der Trennung wird auch die Mutter unvorhersehbarem Stress ausgesetzt. Diese chronische traumatische Exposition während der ersten zwei Lebenswochen hinterlässt bei den Nachkommen langanhaltende Effekte auf die Verhaltensreaktion, von denen einige über mehrere Generationen an die Nachkommen weitergegeben werden. F2-, F3- und in einigen Fällen F4-MSUS-Mäuse zeigen depressionsähnliche Verhaltensweisen, veränderte Reaktionen auf widrige Umweltbedingungen, ein risikofreudigeres Verhalten und beeinträchtigte soziale Fähigkeiten (Franklin et al., 2010; Franklin et al., 2011). F2Tiere haben auch kognitive Defizite, die mit Veränderungen der synaptischen Plastizität (also der Verbindungen von Nervenzellen untereinander und der Reizübertragung) sowie der serotonergen Schaltkreise (also der hormonellen Steuerung) einhergehen (Bohacek et al., 2015; Razoux et al., 2017). Neben dem Verhalten ist auch der Glukoseund Fettstoffwechsel der Nachkommen durch MSUS verändert (van Steenwyk et al., 2020). 
Widrigkeiten im Erwachsenenleben können auch zu Verhaltensdefiziten führen, die auf die Nachkommen übertragen werden können. Chronischer Stress, der durch die Störung der sozialen Hierarchie bei erwachsenen Mäusen ausgelöst wird, verändert das Sozialverhalten über drei Generationen hinweg (Saavedra-Rodríguez/Feig, 2013). Chronische soziale Niederlagen können auch depressive Verhaltensweisen bei den Nachkommen (F1) von gestressten männlichen Mäusen (F0) nach sich ziehen. Einige dieser Effekte konnten durch In-vitro-Fertilisation (IVF) übertragen werden, was auf ihre Abhängigkeit von Keimzellen hindeutet (Dietz et al., 2011). In ähnlicher Weise konnte die Traumatisierung von Mäusen, indem sie unerwünschten Gerüchen ausgesetzt wurden, eine Verhaltensempfindlichkeit gegenüber dem Geruch in ihren F2- und F3-Nachkommen auslösen, die von Veränderungen in ihrem olfaktorischen (den Geruchssinn betreffenden) System begleitet wurde und Keimbahnabhängigkeit zeigte (Dias/Ressler, 2013). Zusammengenommen deuten diese Ergebnisse stark auf eine keimbahnabhängige Übertragung der Auswirkungen von Traumata über Generationen hinweg hin.

\subsubsection{Humanstudien}

Die Weitergabe der Auswirkungen von Traumata über Generationen hinweg beim Menschen ist schon lange Gegenstand von Interesse. Aber erst in jüngster Zeit wird eine epigenetische Grundlage einer solchen Übertragung in Betracht gezogen (siehe Rheinberger/Müller-Wille, Kap. 11, sowie zur skeptischen Gegenposition Alex/Winkler, Kap. 13). Aufgrund des Mangels an kausalen Zusammenhängen und mechanistischen Erklärungen wurden jedoch viele Spekulationen über die epigenetische Vererbung beim Menschen angestellt (Jawaid et al., 2018; Jawaid et al., 2021).

Wie bei Tieren scheint auch beim Menschen ein frühes Trauma eines Elternteils ein entscheidender Faktor für das Verhalten der Nachkommen zu sein. Geringe mütterliche Bindung und mütterlicher Stress wurden in einer deutschen Studie mit einer Borderline-Persönlichkeitsstörung bei den Kindern von betroffenen Personen in Verbindung gebracht (Infurna et al., 2016). Eine schwedische Studie zeigte, dass der elterliche Tod während der Vorpubertät von Jungen mit Frühgeburtlichkeit und niedrigem Geburtsgewicht ihrer Kinder verbunden war (Vågerö/Rajaleid, 2017).

Die Erfahrung von Völkermord und Gewalt ist ein weiterer Auslöser für eine mögliche Übertragung von Psychopathologien auf die Kinder traumatisierter Personen. Einige ältere Berichte wiesen auf erhöhte psychiatrische Erkrankungen bei Kindern von Holocaust-Überlebenden hin (Barocas/Barocas, 1979). Studien aus der Gruppe von Rachel Yehuda haben deutliche neuropsychiatrische Beeinträchtigungen bei den Nachkommen und Enkeln von Holocaust-Überlebenden gezeigt und erste Hinweise darauf 
geliefert, dass dies mit einer Veränderung der DNA-Methylierung in einem bestimmten Gen (FK506-Bindungsprotein 5) einhergeht, das beim Menschen die Empfindlichkeit gegenüber Stresshormonen reguliert (Yehuda et al., 2016). Die Nachkommen von Holocaust-Überlebenden, insbesondere von weiblichen Überlebenden, zeigen eine erhöhte Anfälligkeit für eine posttraumatische Belastungsstörung (PTBS) und haben zu Beginn der Studie niedrigere Werte des Stresshormons Cortisol, ein Effekt, der bereits bei PTBS beobachtet wurde (Yehuda et al., 1998; Yehuda et al., 2000; Yehuda et al., 2008). Die elterliche Exposition gegenüber dem Völkermord in Kambodscha und Ruanda wurde mit erhöhter Angst und Depression bei den Nachkommen in Verbindung gebracht (Field et al., 2013; Rieder/Elbert, 2013).

Neben Völkermord ist Vertreibung eine weitere traumatische Erfahrung, die mit der intergenerationalen Vererbung von Verhaltensstörungen beim Menschen verbunden sein könnte. Kinder von Personen, die nach Zwangsvertreibung eine PTBS entwickelten, zeigen eine implizite Vermeidung von vertreibungsbezogenen Reizen wie emotionalen Wörtern, z. B. Krieg, Tod (sog. emotionale Stroop-Aufgabe) (Wittekind et al., 2010). In ähnlicher Weise wurden bei lateinamerikanischen Einwanderern in die USA einige Symptome von traumatischem Stress an die Nachkommen weitergegeben (Phipps/Degges-White, 2014). Eine schwedische Studie kam zu dem Ergebnis, dass Kinder von Einwanderern, die Folter ausgesetzt waren, ebenfalls vermehrt depressive und somatisierende (psychische Ursachen habende körperliche) Symptome aufweisen (Daud et al., 2005). Eine neuere Studie über die Kinder von finnischen Evakuierten während des Zweiten Weltkriegs zeigte ein erhöhtes Risiko für psychiatrische Krankenhausaufenthalte bei Frauen, deren Mütter evakuiert wurden, im Vergleich zu denen, deren Mütter nicht evakuiert wurden (Santavirta et al., 2018).

Auch ein Kriegstrauma wurde als ein wichtiger Stressfaktor vorgeschlagen, der zu vererbbaren Verhaltensstörungen beim Menschen führt. Die Nachkommen von Veteranen des Zweiten Weltkriegs (Rosenheck, 1986) und des Vietnamkriegs (Glenn et al., 2002) zeigen Gewalttätigkeit und Feindseligkeit, die eng mit der Intensität und Dauer der Erfahrung von Kriegstraumata bei ihren Eltern korrelieren. In ähnlicher Weise zeigen Kinder von Veteranen des serbisch-bosnischen Konflikts eine Vielzahl von Entwicklungs-, Verhaltens- und emotionalen Problemen (Klaric et al., 2008). Darüber hinaus zeigte eine aktuelle Studie über die Nachkommen von Kriegsgefangenen des amerikanischen Bürgerkriegs eine erhöhte Sterblichkeit bei den männlichen Nachkommen (Costa et al., 2018).

Während es schwierig ist, mechanistische Beweise für die Keimbahnabhängigkeit der Übertragung der Auswirkungen von Traumata beim Menschen zu liefern, sind zwei Studien bemerkenswert, die zeigen, dass das Keimbahnepigenom durch Traumaexposi- 
tion beim Menschen verändert werden kann. Die erste Studie lieferte zum ersten Mal den Beweis, dass bestimmte miRNAs im Sperma von Männern verändert sind, die höhere Punktzahlen in einem Fragebogen zu negativen Kindheitserfahrungen aufwiesen (Dickson et al., 2018). Die zweite Studie ist unsere laufende Untersuchung von epigenetischen Markern in Körperflüssigkeiten von Kindern, die ihren Vater verloren haben und von der Mutter getrennt in einem Waisenhaus leben. Dabei zeigten sich Veränderungen in einer Reihe von Serum-miRNAs, von denen bekannt ist, dass sie in die Verhaltensund Stoffwechselregulation involviert sind (Jawaid et al., 2021).

Mit der rasch anwachsenden Zahl an epidemiologischen Studien und jüngsten Nachweisen, die epigenetische Veränderungen in Körperflüssigkeiten und Sperma von traumaexponierten Bevölkerungsgruppen zeigen, wird es zunehmend plausibel, dass Auswirkungen von Trauma beim Menschen übertragbar sind. Dies bedeutet, dass die Auswirkungen des Traumas von exponierten Eltern auf die Kinder übertragen werden können. In dem Fall ist es wichtig zu überlegen, welches die potenziellen Mediatoren sind, die die Auswirkungen des Traumas in die Keimbahn tragen.

\subsection{Die Rolle des Blutes bei der Übertragung der Auswirkungen von Traumaexposition und Krankheitsanfälligkeit}

Während Veränderungen im Epigenom der Keimbahn als Vektoren (Überträger) für die Vererbung der Auswirkungen von Traumata vorgeschlagen wurden, bleibt unbekannt, wie Auswirkungen von Traumata, die im Gehirn aktiviert werden, in reproduktive Zellen getragen werden. Eine entscheidende Rolle könnten hier lösliche Faktoren im Blut spielen, die mit Zellen in den Keimdrüsen kommunizieren könnten. Diese Faktoren werden möglicherweise vom Gehirn oder anderen Organen in Reaktion auf Stress freigesetzt und wirken über Rezeptoren wie adrenerge (Adrenalin enthaltende oder darauf reagierende) Rezeptoren, MR (Mineralocorticoid Receptor), Gonadotropin-ReleasingHormon(GnRH)-Rezeptoren oder Arginin-Vasopressin-Rezeptoren, die auf Keimzellen vorhanden sind (Adeoya-Osiguwa et al., 2006; Fiore et al., 2006; Naz, 2006; Kwon et al., 2013).

Unter diesen zirkulierenden Faktoren könnten Hormone eine Rolle spielen, da bekannt ist, dass ein Trauma das zirkulierende hormonelle Milieu verändert, und gezeigt wurde, dass eine Hormonbehandlung den ncRNA-Gehalt von Spermien verändert (Short et al., 2016). Zytokine (Wachstum und Zelldifferenzierung regulierende Proteine) stellen weitere wichtige zirkulierende Faktoren dar, die zu berücksichtigen sind, da sie 
die Barrieren im Körper regulieren können (Pan et al., 2011). Zytokinrezeptoren können auch auf Keimzellen nachgewiesen werden (Naz, 2006).

Die prominentesten Kandidaten für die Übertragung von Traumasignaturen auf die Keimbahn könnten unserer Meinung nach jedoch zirkulierende RNAs und Stoffwechselprodukte (Metabolite) sein. Zirkulierende RNAs, insbesondere ncRNAs, sind in verschiedenen Körperflüssigkeiten biologisch aktiv und werden entweder verpackt in extrazellulären Vesikeln (EVs) oder in Verbindung mit Lipoproteinen oder Argonaute-Proteinen transportiert. Interessanterweise ist der Fettstoffwechsel, der in vielen Transgenerationsmodellen konsistent verändert ist, eng mit Lipoproteinen sowie mit extrazellulären Vesikeln verbunden. Zwei Drittel aller solcher Vesikel stammen bei Säugetieren aus dem Fettgewebe (Thomou et al., 2017), während Lipoproteinmoleküle bekanntermaßen RNAs tragen (Vickers et al., 2011). Darüber hinaus zeigt unsere laufende Studie bei Mäusen und Menschen vergleichbare Veränderungen in Lipoproteinen und zirkulierenden ncRNAs nach einem frühen Lebenstrauma (Jawaid et al., 2021).

Ein weiterer wichtiger Überträger von Signalen an die Keimbahn könnten zirkulierende Stoffwechselprodukte sein. Eine sich bemerkenswerterweise überschneidende Anzahl an zirkulierenden Stoffwechselprodukten ist bei Mäusen und Menschen nach einem frühen Lebenstrauma verändert. Wichtig ist, dass diese Metaboliten die nukleäre (im Kern stattfindende) Rezeptorsignalübertragung in den Keimzellen verändern können (van Steenwyk et al., 2020).

Eine mögliche Rolle von Blut als Übertragungsmedium für die Auswirkungen von Traumata auf die Keimbahn hat wichtige Implikationen für die Übertragung von Krankheitsanfälligkeit beim Menschen. Darüber hinaus ergeben sich daraus auch potenzielle Möglichkeiten zur Intervention, da Überträger im Blut für Diagnostik und Therapeutik anvisiert werden können. Dies ist auch relevant für die Betrachtung möglicher Implikationen der epigenetischen Übertragung der Auswirkungen von Traumata auf die Gesellschaft.

\subsection{Zur Bedeutung der epigenetischen Vererbungsforschung für die Gesellschaft}

Die Vorstellung, dass traumatische Erfahrungen lang anhaltende Folgen für die geistige und körperliche Gesundheit und das Wohlbefinden der betroffenen Menschen und möglicherweise ihrer Kinder und Enkelkinder haben können, hat weitreichende Folgen für die menschliche Zivilisation und Gesellschaft. Zahlreiche religiöse, politische und ethnische Konflikte haben in den letzten hundert Jahren alle Ecken der Welt heimgesucht, und das Bild ist auch heute noch düster, mit gewaltsamen Konflikten, die viele 
Länder betreffen, von Afghanistan bis Kamerun, Nigeria bis Palästina, Pakistan bis Syrien, Ukraine bis Jemen. Neben den Folgen für die lokale Zivilbevölkerung sind auch Militärangehörige und ihre Familien chronisch Traumata ausgesetzt. Abgesehen von direkten Kriegseinsätzen können auch Vertreibung, erzwungene Familientrennung, ethnische Gewalt und Naturkatastrophen traumatisch sein. Neuere Forschungen haben epigenetische Veränderungen bei Menschen identifiziert, die Völkermorden in Afrika oder dem Holocaust ausgesetzt waren, aber unser Wissen ist noch begrenzt und es wurden keine Studien zu gegenwärtigen Konflikten durchgeführt. Bemühungen um Präventiv- und Abhilfemaßnahmen in betroffenen Bevölkerungsgruppen sind ebenfalls begrenzt, und es gibt keine routinemäßige psychologische oder metabolische Beurteilung der betroffenen Kinder.

Um das Potenzial der präklinischen Forschung an Tiermodellen für ein besseres Verständnis der medizinischen Auswirkungen menschlicher Konflikte zu veranschaulichen, nehmen wir das Beispiel einer aktuell schwierigen Situation in Nordamerika: die Familientrennungskrise an der Grenze zwischen den USA und Mexiko im Jahr 2018, die für viele Familien verheerende Lebensumstände geschaffen hat. In wissenschaftlichen Kreisen wurde Besorgnis über die langfristigen Folgen einer solchen Inhaftierung auf die psychische und physische Gesundheit der der Trennung ausgesetzten Kinder geäußert (Danaher, 2018). Diese Situation hatte viele Ähnlichkeiten mit einem Mausmodell für frühes Trauma, das eine Vererbung von Verhaltens- und Stoffwechselsymptomen über Generationen hinweg zeigt (das MSUS-Modell). Dieses Modell legt nahe, dass es lebenslange Folgen der elterlichen Trennung und Inhaftierung nicht nur für diese Kinder, sondern auch für ihre Nachkommen geben könnte. Wenn man die Erkenntnisse aus solchen Mausstudien auf die Situation der inhaftierten Kinder überträgt, kann man davon ausgehen, dass deren Kinder oder sogar Enkelkinder im Erwachsenenalter ein Risiko für Stoffwechselstörungen und Psychopathologien wie Depressionen und erhöhte Risikobereitschaft haben könnten, die ihr Leben beeinträchtigen würden. Wir selbst haben kürzlich eine Studie an Kindern durchgeführt, die in SoS-Kinderdörfern in Pakistan leben. Diese Kinder haben ein Trauma in Form von Verlust des Vaters und Trennung von der Mutter erlebt und weisen erhöhte Ängstlichkeit, pro-depressive Symptome und Aufmerksamkeitsdefizite sowie verminderte High-Density-Lipoproteine im Blut auf. Darüber hinaus sind einige miRNAs, von denen bekannt ist, dass sie im Serum von Mäusen durch frühe Traumata verändert werden, im Serum dieser Kinder in ähnlicher Weise verändert, was darauf hindeutet, dass molekulare Signalwege, die mit dem Fettstoffwechsel und nicht-kodierenden RNAs in Verbindung stehen, bei Traumata in Mäusen und Menschen erhalten bleiben (Jawaid et al., 2021). Die weitreichenden Verhaltens-, Stoffwechsel- und molekularen Störungen bei diesen Kindern lassen 
vermuten, dass sie in ihrem Erwachsenenleben oder sogar ihre Nachkommen eine Anfälligkeit für zahlreiche gesundheitliche Komplikationen aufweisen könnten. In der Tat führt die Anzüchtung von keimzellähnlichen Zellen in Kultur mit dem Serum dieser Kinder zu einer Übertragung einiger molekularer Veränderungen auf die angezüchteten Zellen, was die Möglichkeit nahelegt, dass das Erbe ihrer schmerzhaften Erfahrungen bestehen bleibt (Curry, 2019).

Die biologischen Wege, über die die Auswirkungen eines Traumas von den Eltern auf die Kinder übertragen werden können, sind nur teilweise verstanden. Während Keimzellen wahrscheinlich dazu beitragen, da sie epigenetische Veränderungen aufweisen, die an die Nachkommen weitergegeben werden, können andere biologische Substanzen wie Körperflüssigkeiten wahrscheinlich ebenfalls dazu beitragen. Die Muttermilch während der Stillzeit ist ein Kandidat. Studien an Mäusen, Primaten und Menschen haben gezeigt, dass Mütter während der Stillzeit biologisch aktive Stoffe übertragen, die die Gehirnentwicklung, das Verhalten und das Temperament ihrer Nachkommen beeinflussen können (Liu et al., 2014; Hinde et al., 2015). Dies könnte für schwangere und stillende Mütter in Konfliktregionen und für Frauen, die in friedenssichernden Kräften dienen, von Bedeutung sein.

Obwohl eine Traumaexposition insgesamt schädlich für die betroffenen Individuen ist, kann sie unter bestimmten Bedingungen zur Entwicklung von adaptiven Verhaltensweisen führen, die für sie und ihre Nachkommen von Vorteil sind. Bei Mäusen gibt es Belege dafür, dass adaptive Verhaltensweisen wie verbesserte Verhaltensflexibilität und zielgerichtete Reaktionen bei traumaexponierten Individuen und ihren Nachkommen auftreten und ihnen helfen können, besser mit herausfordernden Situationen umzugehen (Gapp et al., 2014b). Ebenso hat eine Studie am Menschen gezeigt, dass Enkelkinder von Immigrant*innen bessere akademische Leistungen haben als Kinder der dritten oder vierten Generation (Duong et al., 2016). Es sind jedoch weitere Studien der Mechanismen erforderlich, um die Wege zu identifizieren, die die Übertragung resilienter (widerstandsfähiger) Verhaltensweisen auf die Nachkommenschaft bewirken, und um herauszufinden, ob irgendeine Maßnahme eine solche „positive Übertragung“ nach einer Traumaexposition begünstigen könnte. Von besonderer Bedeutung ist der optimale Zeitpunkt für eine effiziente Intervention.

\subsection{Günstige Zeitfenster}

Mit den neuen Erkenntnissen über die durch Trauma veränderten molekularen Signalwege und die potenziellen Mechanismen für deren Vererbung ist es nun möglich, Verhaltensinterventionen, Nahrungsergänzung und/oder pharmakologische Inter- 
ventionen bei traumaexponierten Bevölkerungsgruppen in Betracht zu ziehen. Tierstudien deuten darauf hin, dass die Übertragung einiger Traumafolgen auf die Nachkommen verhindert wird, wenn traumabetroffene Männchen vor der Empfängnis in ein reichhaltigeres Lebensumfeld (z. B. einen großen Käfig mit Spielzeug, Laufrädern und einem Labyrinth) versetzt werden (Gapp et al., 2016). Darüber hinaus können einige Gehirnmoleküle, von denen bekannt ist, dass sie die soziale Bindung erhöhen, wie z. B. Oxytocin und Vasopressin, die intergenerationalen Effekte von chronischem sozialen Stress bei Mäusen rückgängig machen. Weibliche F2-Nachkommen von Müttern, die solchem Stress ausgesetzt waren, haben erhöhte sich wiederholende Handlungen und Denkstörungen und ängstliche Verhaltensweisen sowie Defizite im Sozialverhalten. Die Behandlung von F1-Muttertieren mit Oxytocin nach chronischem sozialem Stress verhindert die Weitergabe von repetitivem und ängstlichem Verhalten an die F2-Nachkommen, während die intranasale Vasopressin-Behandlung bei F1-Muttertieren positive Auswirkungen auf Verhaltensdefizite und Ängstlichkeit zeigte (Murgatroyd et al., 2016).

Trotz dieser begrenzten Evidenz geben diese Studien Anlass zur Hoffnung, dass die Übertragung von Traumasymptomen beim Menschen teilweise vermeidbar sein könnte. Die Herausforderung besteht darin, solche ermutigenden Aspekte aus Tierstudien zu nutzen, um klinische und soziale Interventionen zu entwerfen, die die Übertragung von Traumasymptomen beim Menschen verhindern. Um sich dies besser vorstellen zu können und mögliche Zeitfenster für Interventionen zu identifizieren, werden wir das hypothetische Beispiel von Kriegswaisen aus einer Konfliktregion ohne elterliche Fürsorge, die chronisch widrigen Bedingungen ausgesetzt sind, verwenden. Basierend auf unserer Arbeit an Mäusen und Menschen mit frühkindlichen psychologischen Traumata lässt sich vorhersagen, dass ein betroffenes Kind eine größere Anfälligkeit für psychische und körperliche Störungen zeigt und diese Anfälligkeiten schließlich an seine eigenen Kinder weitergeben könnte.

Möglicherweise hilfreiche Interventionen umfassen:

1. Ein Ansatzpunkt für eine Intervention könnte ein psychologisches Screening des Kindes sein, gefolgt von einer geeigneten psychologischen Therapie und Nahrungsergänzung. Die psychologische Intervention könnte eine multimodale (auf vielfältige Art und Weise erfolgende) Stimulation beinhalten, ein Äquivalent zu besseren Lebensbedingungen (Environmental Enrichment), die bei Nagetieren eingesetzt werden.

2. Laufende Screenings und Interventionen könnten hilfreich sein, wenn das Kind erwachsen wird und eine Elternschaft plant. Die Screenings könnten Keimbahn- 
untersuchungen auf epigenetische Signaturen von Traumata beinhalten, von denen bekannt ist, dass sie im Erwachsenenalter fortbestehen und mit spezifischen Störungsbildern der Phänotypen bei den Nachkommen in Verbindung gebracht werden.

3. Die Nachkommen der anfänglichen Kriegswaisen könnten dann auf atypische Verhaltensweisen und neuropsychiatrische und/oder metabolische Anomalien untersucht werden, die aus der epigenetischen Analyse der elterlichen Keimbahn vorhergesagt werden könnten, und es könnte ihnen, wo nötig, eine angemessene Behandlung angeboten werden.

Es ist bemerkenswert, dass keine dieser Interventionen bisher am Menschen getestet wurde, weshalb eine sorgfältige Beurteilung und Bewertung erforderlich ist, bevor sie in der Praxis umgesetzt werden können. Die Umsetzung solcher Interventionen könnte mit zahlreichen Herausforderungen verbunden sein, angefangen bei der Frage, welche Plattform (Methode) für ein breit angelegtes Screening psychischer Beeinträchtigungen bei traumatisierten Personen verwendet werden soll, bis hin zu ethischen Erwägungen im Zusammenhang mit der Bewertung von Keimbahnuntersuchungen. Die moralischen und sozialen Implikationen solcher Eingriffe und die Verantwortung jedes Einzelnen für seine eigene Gesundheit und die Gesundheit seiner Nachkommen sind wichtige Fragen, die einer Reflexion bedürfen. Organisationen, die auf globaler Ebene arbeiten, wie die Weltgesundheitsorganisation (WHO), die Vereinten Nationen, das Kinderhilfswerk der Vereinten Nationen (UNICEF) und das Flüchtlingshilfswerk der Vereinten Nationen (UNHCR), könnten eine wichtige Rolle bei der Befürwortung und Umsetzung dieser Interventionen in instabilen und konfliktträchtigen Regionen spielen. Vorstellbar ist, dass zukünftig im klinischen Umfeld ein strenges Screening für Trauma anhand von psychologischen und neu entstehenden molekularen Biomarkern eingesetzt wird, um die Langzeitfolgen in gefährdeten Gemeinschaften, wie z. B. bei Immigrant*innen, vorherzusagen. Anschließend könnte man Personen, die epigenetische Signaturen der Traumaexposition tragen, sowie ihren Nachkommen in Zukunft Beratung, Prophylaxe und/oder Therapie anbieten (Jawaid et al., 2021).

Einige bemerkenswerte Beispiele für soziale Initiativen, die sich als hilfreich erweisen könnten, um die Ausbreitung von Traumasymptomen bei Menschen zu verhindern und/oder zu reduzieren, bieten die Empfehlungen des Europarats für Einwandererpopulationen ${ }^{4}$ und die Beratungsinitiative für Genesung und Wohlbefinden des Minis-

4 Siehe unter: https://www.coe.int/t/democracy/migration/ressources/recommendationsresolutions_en.asp [12.05.2021]. 
teriums für Jugendentwicklung in Neuseeland, ${ }^{5}$ die nach dem Anschlag in Christchurch 2019 ins Leben gerufen wurde. Die Programme nutzen auf positiver Psychologie basierende Maßnahmen, um die Integration von Flüchtlingen zu verbessern bzw. Menschen bei der Bewältigung von Traumata zu helfen. Ein wichtiger Aspekt könnte hier die Einbeziehung der Bewertung von epigenetischen Traumamarkern in Körperflüssigkeiten (Blut, Speichel etc.) sein, die durch die Zeit gemessen werden könnten, um zu testen, ob solche Interventionen die epigenetischen Veränderungen aufgrund von Traumaexposition reduzieren können.

Neben der Vorbeugung oder Verringerung der Folgen von Traumaexposition können die Erkenntnisse aus der epigenetischen Forschung genutzt werden, um die Menschenrechte zu fördern und die Faktoren, die zu Trauma und Viktimisierung führen, zu verringern. Beim Menschen liegt Gewalt oft in der Familie (Black et al., 2010), was darauf hindeutet, dass gewalttätiges oder ausgrenzendes Verhalten auch genetisch oder epigenetisch vererbt werden kann. Wenn dies zutrifft kann angesichts dessen, dass epigenetische Faktoren modifizierbar sind, die Möglichkeit in Betracht gezogen werden, die Übertragung solcher Verhaltensweisen durch Einwirkung auf das Epigenom zu mildern. ${ }^{6}$ Beispielsweise könnte versucht werden, die Auswirkungen von Traumata auf Gehirn und Körper durch Interventionen auf Basis von virtueller Realität (VR) zu verbessern. Es könnte auch in Konfliktsituationen in gewalttätigen Gemeinschaften nützlich sein, um das Einfühlungsvermögen von Führungspersönlichkeiten in Gemeinschaften zu erhöhen und/oder ihre negativen Vorurteile gegenüber Personen anderer Ethnien abzubauen (Segal et al., 2017). Eine andere Idee könnte sein, VR-Module zu konstruieren, in denen Personen virtuell miterleben können, wie sie selbst und ihre Familienmitglieder einer rassifizierten Menschengruppe angehören, gegen die sie sonst Vorbehalte haben. Es wird interessant sein zu sehen, ob solche Interventionen erfolgreich sind, zu langfristigen epigenetischen Veränderungen führen und möglicherweise vererbbare positive Effekte haben. Dies ist sehr wahrscheinlich, da epigenetische Faktoren durch zelluläre und Gehirnaktivität modifizierbar sind. Es liegt in der Verantwortung der wissenschaftlichen Gemeinschaft, damit zu beginnen, die Machbarkeit und Anwendbarkeit solcher Interventionen zur Förderung der Menschenrechte zu bewerten.

5 Siehe unter: https://www.myd.govt.nz/resources-and-reports/recovery-and-wellbeing-advice. html [12.05.2021].

6 Siehe unter: https://onlinelibrary.wiley.com/doi/10.1002/ajmg.b.32388 [12.05.2021]. 


\subsection{Schlussfolgerungen und Ausblick}

Die wissenschaftliche Forschung des letzten Jahrzehnts hat gezeigt, dass die Folgen von Traumaexposition vererbbar sein können. Es wurde begonnen, die Rolle epigenetischer Faktoren als potenzielle Vermittler solcher Übertragungen und die molekularen Komponenten, die für ihre Induktion in Keimzellen verantwortlich sind, zu untersuchen. Aber viele Fragen und Herausforderungen sind auf diesem Gebiet noch nicht geklärt. Erstens müssen die Art, der Schweregrad und das Ausmaß der Symptome bei den Nachkommen von Personen, die einem Trauma ausgesetzt waren, sorgfältig untersucht werden, da sie wahrscheinlich nicht nur psychische, sondern auch körperliche Störungen umfassen. Zweitens müssen individuelle Unterschiede in der Ausprägung und Übertragung von Symptomen sorgfältig untersucht werden. Zunehmende Hinweise aus Humanstudien deuten darauf hin, dass nur ein Bruchteil der Individuen nach einer Traumaexposition eine PTBS entwickelt, während einige wenige Individuen resilienter werden (ein Phänomen, das als posttraumatisches Wachstum beschrieben wird [Horn et al., 2016]). Es ist möglich, dass die übertragenen Eigenschaften bei traumasensiblen und resilienten Individuen unterschiedlich sind. Drittens sollten zugängliche biologische Flüssigkeiten wie Speichel, Blut und Sperma bei traumatisierten Menschen und idealerweise auch bei deren Nachkommen analysiert werden, um festzustellen, ob die in Tiermodellen beobachteten Veränderungen auch beim Menschen vorkommen.

Schließlich empfehlen wir die Bildung von Think Tanks, die Friedensaktivist*innen, Biolog*innen, Kliniker*innen und Soziolog*innen zusammenbringen, um wissenschaftliche Erkenntnisse in konkrete langfristige humanitäre Bemühungen und politische Rahmenbedingungen umzusetzen. Es ist auch dringend notwendig, dass Friedens- und Menschenrechtsaktivist*innen die verfügbaren Foren nutzen, um die Öffentlichkeit über die Auswirkungen von Traumata über Generationen hinweg zu informieren und so die Öffentlichkeit für das Problem zu sensibilisieren. Jede/r, die/der Zeug*in eines traumatisierten Kindes wird, sollte wissen, dass Trauma andauert, sich ausbreitet, den ganzen Körper betrifft und übertragbar ist. Der einzige bekannte Weg, um zu verhindern, dass Generationen von Opfern unter einem Trauma leiden, ist der Aufbau einer Gesellschaft, die auf Frieden basiert.

\section{Danksagung}

Wir danken den Mitgliedern des Labors für Neuroepigenetik (Universität Zürich/ETH Zürich) für konstruktives Feedback zum Entwurf dieses Artikels. 


\subsection{Literaturverzeichnis}

Adeoya-Osiguwa, S. A. et al. (2006): Identification of functional Alpha2- and Beta-Adrenergic receptors in mammalian spermatozoa. In: Human Reproduction 21(6): 1555-1563.

Babb, J. A. et al. (2014): Transgenerational effects of social stress on social behavior, corticosterone, oxytocin, and prolactin in rats. In: Hormones and Behavior 65(4): 386-393.

Bahar Halpern, K. et al. (2014): Paradoxical role of DNA methylation in activation of FoxA2 gene expression during endoderm development. In: Journal of Biological Chemistry 289(34): 23882-23892.

Bannister, A. J./Kouzarides, T. (2011): Regulation of chromatin by histone modifications. In: Cell Research 21(3): 381-395.

Bird, A. (2002): DNA methylation patterns and epigenetic memory. In: Genes and Development 16(1): 6-21.

Black, D. S. et al. (2010): A further look at the intergenerational transmission of violence: Witnessing interparental violence in emerging adulthood. In: Journal of Interpersonal Violence 25: 1022-1042.

Bohacek, J./Mansuy, I. M. (2015): Molecular insights into transgenerational non-genetic inheritance of acquired behaviours. In: Nature Reviews Genetics 16(11): 641-652.

Bohacek, J. et al. (2015): Pathological brain plasticity and cognition in the offspring of males subjected to postnatal traumatic stress. In: Molecular Psychiatry 20: 621-631.

Cao, J./Yan, Q. (2012): Histone ubiquitination and deubiquitination in transcription, DNA damage response, and cancer. In: Frontiers in Oncology 2: 26.

Cheng, J. et al. (2014): SUMOylation of MeCP2 is essential for transcriptional repression and hippocampal synapse development. In: Journal of Neurochemistry 128(6): 798-806.

Ciabrelli, F. et al. (2017): Stable polycomb-dependent transgenerational inheritance of chromatin states in Drosophila. In: Nature Genetics 49(6): 876-886.

Costa, D. L. et al. (2018): Intergenerational transmission of paternal trauma among US Civil War ExPOWs. In: Proceedings of the National Academy of Sciences of the United States 115: 11215-11220.

Curry, A. (2019): A painful legacy. In: Science 365: 212-215.

Danaher, F. (2018): The suffering of children. In: New England Journal of Medicine 379: e4.

Daud, A. et al. (2005): Children in families of torture victims: Transgenerational transmission of parents' traumatic experiences to their children. In: International Journal of Social Welfare 14(1): 23-32.

Dias, B. G./Ressler, K. J. (2013): Parental olfactory experience influences behavior and neural structure in subsequent generations. In: Nature Neuroscience 17(1): 89-96.

Dickson, D. A. et al. (2018): Reduced levels of miRNAs 449 and 34 in sperm of mice and men exposed to early life stress. In: Translational Psychiatry 8: 101.

Dietz, D. M. et al. (2011): Paternal transmission of stress-induced pathologies. In: Biological Psychiatry 70(5): 408-414.

Duong, M. T. et al. (2016): Generational differences in academic achievement among immigrant youths: A meta-analytic review. In: Review of Educational Research 86: 3-41. 
Field, N. P. et al. (2013): Parental styles in the intergenerational transmission of trauma stemming from the Khmer Rouge Regime in Cambodia. In: American Journal of Orthopsychiatry 83(4): 483494.

Fiore, C. et al. (2006): Identification of the mineralocorticoid receptor in human spermatozoa. In: International Journal of Molecular Medicine 18(4): 649-652.

Franklin, T. B. et al. (2010): Epigenetic transmission of the impact of early stress across generations. In: Biological Psychiatry 68(5): 408-415.

Franklin, T. B. et al. (2011): Influence of early stress on social abilities and serotonergic function across generations in mice. In: PLOS ONE 6: 21842.

Gapp, K. et al. (2014a): Implication of sperm RNAs in transgenerational inheritance of the effects of early trauma in mice. In: Nature Neuroscience 17(5): 667-669.

Gapp, K. et al. (2014b): Early life stress in fathers improves behavioural flexibility in their offspring. In: Nature Communications 5: 5466.

Gapp, K. et al. (2016): Potential of environmental enrichment to prevent transgenerational effects of paternal trauma. In: Neuropsychopharmacology 41: 2749-2758.

Gapp, K., et al. (2020): Alterations in sperm long RNA contribute to the epigenetic inheritance of the effects of postnatal trauma. In: Molecular Psychiatry 25: 2162-2174.

Gaydos, L. J. et al. (2014): H3K27me and PRC2 transmit a memory of repression across generations and during development. In: Science 345(6203): 1515-1518.

Ginzburg, K. et al. (2010): Comorbidity of posttraumatic stress disorder, anxiety and depression: A 20-year longitudinal study of war veterans. In: Journal of Affective Disorders 123(1-3): 249-257.

Glenn, D. M. et al. (2002): Violence and hostility among families of Vietnam veterans with combatrelated posttraumatic stress disorder. In: Violence and Victims 17(4): 473-489.

Ha, M./Kim, V. N. (2014): Regulation of MicroRNA biogenesis. In: Nature Reviews Molecular Cell Biology 15(8): 509-524.

Harvey, A. et al. (1979): Wounds of the fathers: The next generation of Holocaust victims. In: International Review of Psycho-Analysis 1: 331-340.

Hinde, K. et al. (2015): Cortisol in mother's milk across lactation reflects maternal life history and predicts infant temperament. In: Behavioral Ecology 26: 269-281.

Horn S.R. et al. (2016): Understanding resilience: New approaches for preventing and treating PTSD. In: Experimental Neurology 284: 119-132.

Hunter, R. G. et al. (2009): Regulation of hippocampal H3 histone methylation by acute and chronic stress. In: Proceedings of the National Academy of Sciences of the United States 106(49): 2091220917.

Infurna, M. R. et al. (2016): Parents' childhood experiences of bonding and parental psychopathology predict borderline personality disorder during adolescence in offspring. In: Psychiatry Research 246: 373-378.

Jawahar, M. C. et al. (2015): Epigenetic alterations following early postnatal stress: A review on novel aetiological mechanisms of common psychiatric disorders. In: Clinical Epigenetics 7(1): 122.

Jawaid, A. et al. (2018): Transgenerational epigenetics of traumatic stress. In: Progress in Molecular Biology and Translational Science 158: 273-298. 
Jawaid, A./Mansuy, I. M. (2019): Inter- and transgenerational inheritance of behavioral phenotypes. In: Current Opinion in Behavioral Sciences 25: 96-101.

Jawaid, A. et al. (2020): Distinct microRNA signature in human serum and germline after childhood trauma. In: MedRxiv. DOI: 10.1101/2020.08.11.20168393.

Jawaid, A. et al. (2021): Impact of parental exposure on offspring health. In: Trends in Genetics 37: 373-388.

Jawaid, A./Mansuy I. M. (2021): Transmission of the effects of trauma across generations: Implications for individuals and society and its role in violent conflict. In: NeuroPeace 3: 5-24. Unter: https://2a362984-4f4f-474d-91d8-f919ce482921.filesusr.com/ugd/f0e071_4d883dc8a168449e8ff1b9aaf5adf2a5.pdf [12.05.2021].

Klaric, M. et al. (2008): Psychological problems in children of war veterans with posttraumatic stress disorder in Bosnia and Herzegovina: Cross-sectional study. In: Croatian Medical Journal 49(4): 491-498.

Klose, R. J./Bird, A. P. (2006): Genomic DNA methylation: The mark and its mediators. In: Trends in Biochemical Sciences 31(2): 89-97.

Kwon, W.-S. et al. (2013): Vasopressin effectively suppresses male fertility. In: PLOS One 8(1): e54192.

Li, B. et al. (2007): The role of chromatin during transcription. In: Cell 128(4): 707-719.

Liu, B. et al. (2014): Maternal hematopoietic TNF, via milk chemokines, programs hippocampal development and memory. In: Nature Neuroscience 17: 97-105.

Lupien, S. J. et al. (2009): Effects of stress throughout the lifespan on the brain, behaviour and cognition. In: Nature Reviews Neuroscience 10(6): 434-445.

Murgatroyd, C. A. et al. (2016): Effects of chronic social stress and maternal intranasal oxytocin and vasopressin on offspring Interferon- $\gamma$ and behavior. In: Frontiers in Endocrinology 7: 155.

Naz, R. K. (2006): Receptors in spermatozoa: Are they real? In: Journal of Andrololgy 27(5): 627-636.

Pan, W. et al. (2011): Cytokine signaling modulates blood-brain barrier function. In: Current Pharmaceutical Design 33: 3729-3740.

Peters, A. H./Schübeler, D. (2005): Methylation of histones: Playing memory with DNA. In: Current Opinion in Cell Biology 17(2): 230-238.

Phipps, R. M./Degges-White, S. (2014): A new look at transgenerational trauma transmission: Secondgeneration Latino immigrant youth. In: Journal of Multicultural Counseling and Development 42(3): 174-187.

Quinn, J. J./Chang, H. Y. (2016): Unique features of long non-coding RNA biogenesis and function. In: Nature Reviews Genetics 17(1): 47-62.

Qureshi, S. U. et al. (2010): Greater prevalence and incidence of dementia in older veterans with posttraumatic stress disorder. In: Journal of the American Geriatric Society 58(9): 1627-1633.

Razoux, F. et al. (2017): Transgenerational disruption of functional 5-HT ${ }^{1 \mathrm{~A}} \mathrm{R}$-induced connectivity in the adult mouse brain by traumatic stress in early life. In: Molecular Psychiatry 22: 519-526. 
Reed, B. et al. (2012): Chromatin alterations in response to forced swimming underlie increased prodynorphin transcription. In: Neuroscience 220: 109-118.

Rieder, H./Elbert, T. (2013): The relationship between organized violence, family violence and mental health: Findings from a community-based survey in Muhanga, Southern Rwanda. In: European Journal of Psychotraumatology 4(1): 21329.

Rodgers, A. B. et al. (2015): Transgenerational epigenetic programming via sperm MicroRNA recapitulates effects of paternal stress. In: Proceedings of the National Academy of Sciences of the United States 112(44): 13699-13704.

Rosenheck, R. (1986): Impact of posttraumatic stress disorder of World War II on the next generation. In: Journal of Nervous and Ment Disease 174(6): 319-327.

Saavedra-Rodríguez, L./Feig, L. A. (2013): Chronic social instability induces anxiety and defective social interactions across generations. In: Biological Psychiatry 73(1): 44-53.

Santavirta, T. et al. (2018): Association of the World War II finnish evacuation of children with psychiatric hospitalization in the next generation. In: JAMA Psychiatry 75(1): 21.

Segal, L. et al. (2017): Promoting human rights through science. In: Science 358: 34-37.

Short, A. K. et al. (2016): Elevated paternal glucocorticoid exposure alters the small noncoding RNA profile in sperm and modifies anxiety and depressive phenotypes in the offspring. In: Translational Psychiatry 6(6): e837.

Snijders, C. et al. (2017): MicroRNAs in post-traumatic stress disorder. In: Current Topics in Behavioral Neurosciences: 1-24.

Thomou, T. et al. (2017): Adipose-derived circulating miRNAs regulate gene expression in other tissues. In: Nature 542: 450-455.

Vågerö, D./Rajaleid, K. (2017): Does childhood trauma influence offspring's birth characteristics? In: International Journal of Epidemiology 46(1): 219-229.

van Steenwyk, G. et al. (2018): Transgenerational inheritance of behavioral and metabolic effects of paternal exposure to traumatic stress in early postnatal life: Evidence in the $4^{\text {th }}$ generation. In: Environmental Epigenetics 4: 023.

van Steenwyk, G. et al. (2020): Involvement of circulating factors in the transmission of paternal experiences through the germline. In: EMBO Journal 39: e104579.

Vickers, K. C. et al. (2011): MicroRNAs are transported in plasma and delivered to recipient cells by high-density Lipoproteins. In: Nature Cell Biology 13: 423-433.

Whittle, N./Singewald, N. (2014): HDAC inhibitors as cognitive enhancers in fear, anxiety and trauma therapy: Where do we stand? In: Biochemical Society Transactions 42(2): 569-581.

Wittekind, C. E. et al. (2010): Intergenerational transmission of biased information processing in posttraumatic stress disorder (PTSD) following displacement after World War II. In: Journal of Anxiety Disorders 24(8): 953-957.

$\mathrm{Wu}$, R. et al. (2016): Transgenerational impairment of hippocampal Akt-mTOR signaling and behavioral deficits in the offspring of mice that experience postpartum depression-like illness. In: Progress in Neuro-Psychopharmacology and Biological Psychiatry 73: 11-18. 
Yehuda, R. et al. (1998): Relationship between posttraumatic stress disorder characteristics of Holocaust survivors and their adult offspring. In: American Journal of Psychiatry 155(6): 841-843.

Yehuda, R. et al. (2000): Low cortisol and risk for PTSD in adult offspring of Holocaust survivors. In: American Journal of Psychiatry 157(8): 1252-1259.

Yehuda, R. et al. (2008): Maternal, not paternal, PTSD is related to increased risk for PTSD in offspring of Holocaust survivors. In: Journal of Psychiatric Research 42(13): 1104-1111.

Yehuda, R. et al. (2016): Holocaust exposure induced intergenerational effects on FKBP5 methylation. In: Biological Psychiatry 80(5): 372-380. 